\title{
Applications of Digital Pathology in the Pharmaceutical Industry
}

\author{
G.D. Gagne, * J.H. Decker,* M. Klapczynski,* W. Buck,** and J.A.Fagerland* \\ * Preclinical Safety Division, Abbott Laboratories, Abbott Park, IL 60064 \\ ** Cellular, Molecular, and Experimental Toxicology, Abbott Laboratories, Abbott Park, IL 60064
}

Advances in digital pathology instrumentation have led to its widespread implementation in pharmaceutical research and development [1,2]. Using high-resolution digital slide scanning systems, whole tissue sections can be rapidly scanned and presented as virtual slides to collaborators worldwide, with image quality approaching that of the glass slide. The development of tools to perform sophisticated analysis at the whole-slide level has led to additional applications of this technology. Recently, we have implemented a digital pathology system at Abbott sites across the U.S and in Germany, and are routinely using it for multisite slide sharing, global pathology teleconferencing, and pathologist-to-pathologist consultation. This presentation will highlight additional uses of digital pathology, such linking ultrastructural pathology to histopathology, quantitation of histopathological changes on whole slide images, and documenting efficiency of laser capture microdissection.

In a repeated dose toxicity study in cynomolgus monkeys, accumulation of pigment was noted by histopathology in liver and adrenal gland. Ultrastructural evaluation was requested to determine the nature of the pigment. Using digital pathology, the pathologist and electron microscopist, at different sites, were able to view and compare the histology slides and semithin sections from EM blocks to assure that the changes seen by histopathology were present in sections taken for EM (Fig. 1). The microscopist was also able to access and view semithin section images while at the electron microscope to aid in locating the desired areas for EM examination.

Liver sections were evaluated to assess vacuolization due to fatty accumulation in hepatocytes from rats dosed orally with a compound for five days. Slides were scanned and quantitation of vacuolization was performed by image analysis of random selections and by automated pattern recognition on whole slide images (Fig. 2). Results from the two methods were comparable (Fig. 2B). The whole slide method had the advantages of unattended analysis and evaluation of the entire tissue section, thus reducing potential sampling bias.

Laser capture microdissection was performed on kidney sections to isolate glomeruli for correlation of gene expression and biomarkers of kidney injury. Slides were scanned before and after LCM (Fig. 3). The before and after images could then be linked and viewed simultaneously to evaluate and document the efficiency and specificity of glomerular capture.

These examples demonstrate digital pathology to be an enabling technology that will continue to find additional uses as technology improves and its adoption becomes more widespread.

References

[1] B. McCullough, et al., Toxicol. Pathol. 32 (Suppl. 2) (2004) 49.

[2] L. Mulrane, et al., Expert. Rev. Mol. Diagn. 8 (2008) 707. 


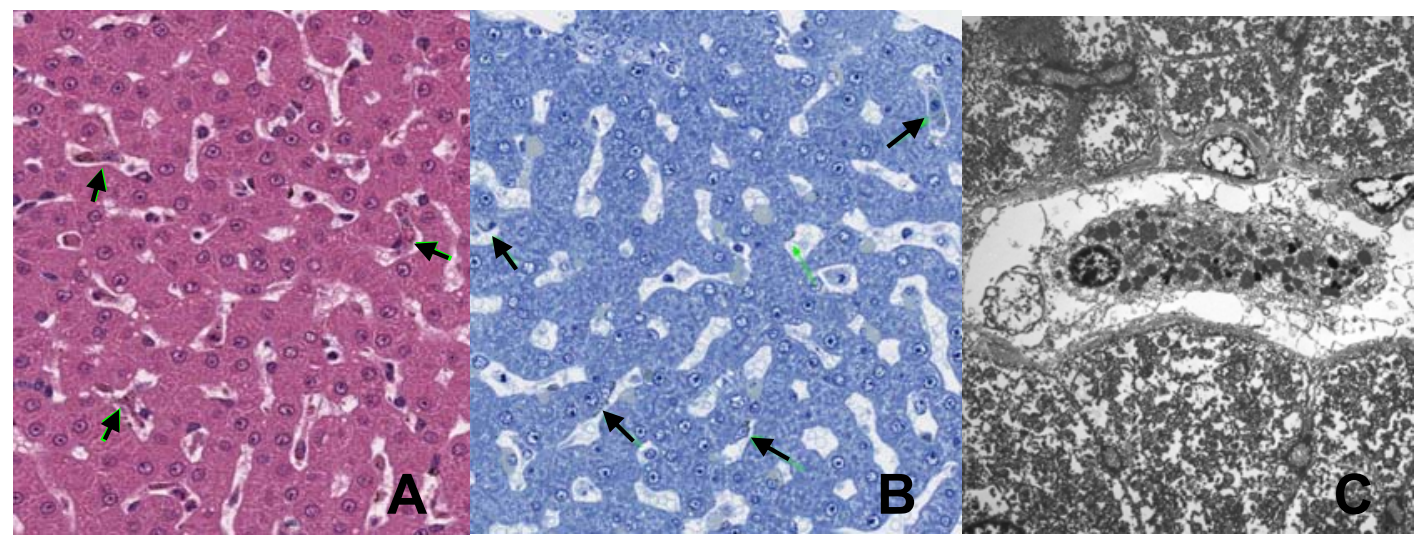

FIG. 1. Paraffin-embedded, H\&E stained liver section (A) and epoxy-embedded semithin section (B) at same magnification. Arrows indicate pigmented cells that were evaluated ultrastructurally (C).
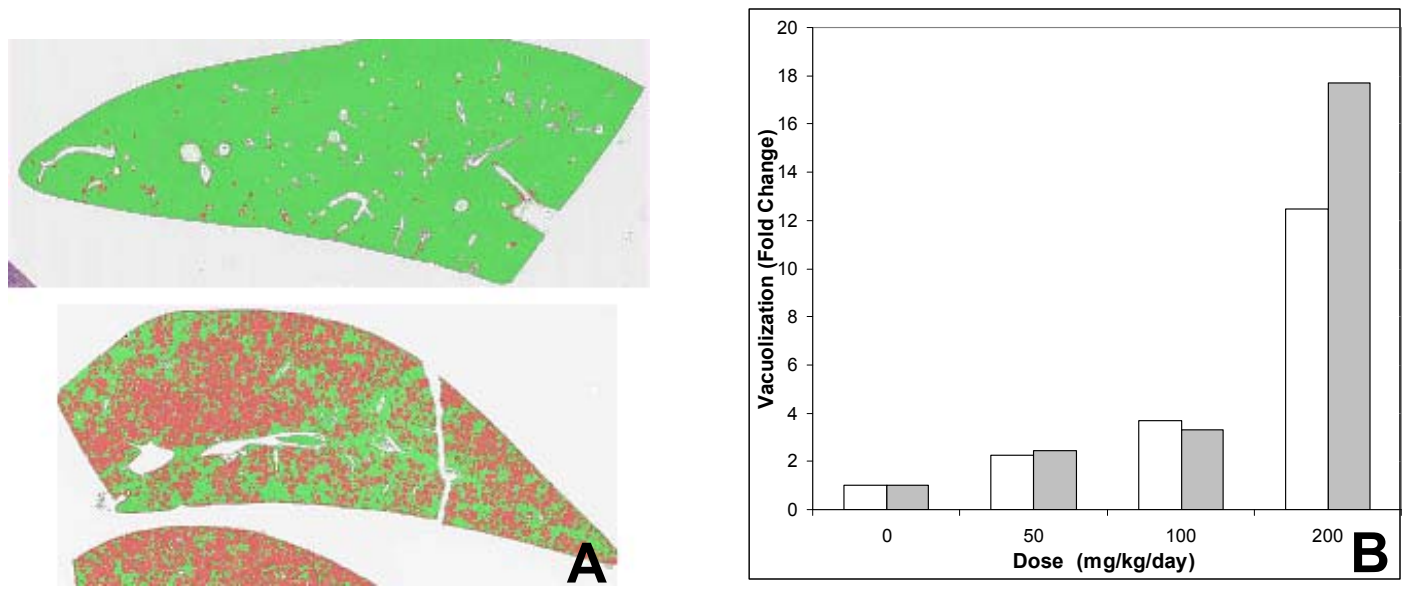

FIG. 2. A-Hepatocyte vacuolation detected on whole-slide images of liver sections by pattern recognition. TOP: Vehicle control. BOTTOM: Dosed rat. Red color indicates regions of vacuolated hepatocytes. B-Quantitation of hepatocyte vacuolation from same sections using threshold-based image analysis (open bars) and automated pattern recognition (shaded bars).

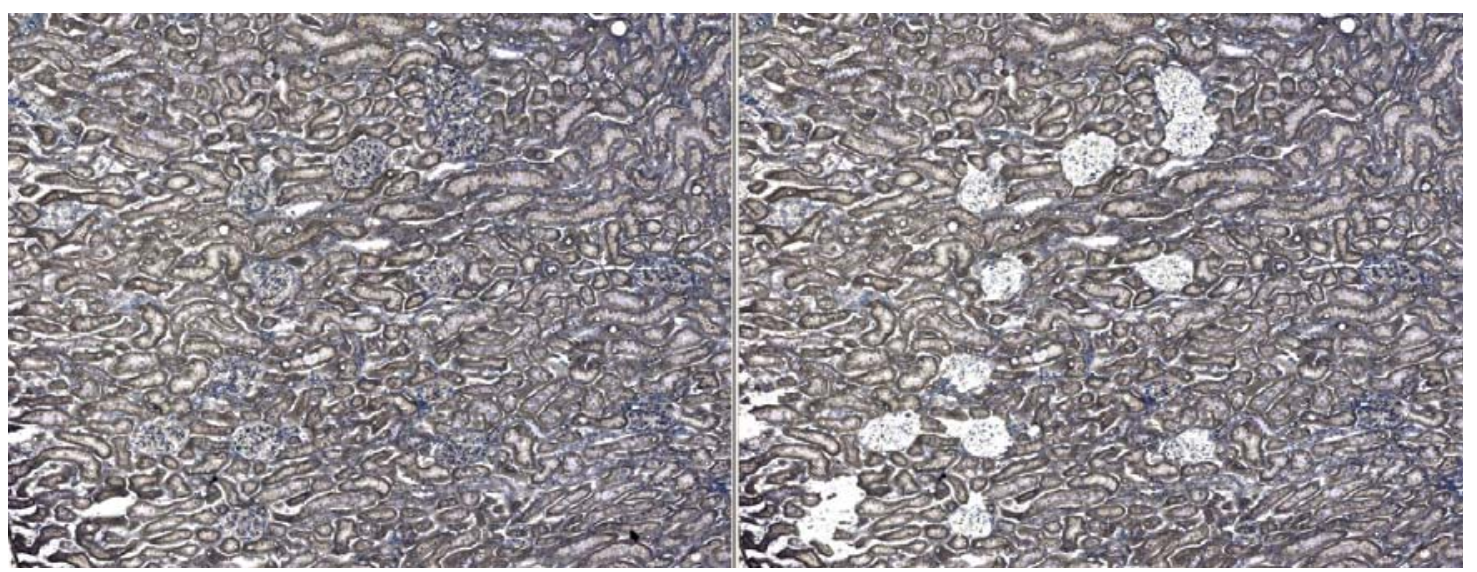

FIG. 3. Kidney section before (left) and after (right) laser capture microdissection of glomeruli. Images were linked and viewed simultaneously to evaluate efficiency of laser capture. 